\title{
LOS MOOCS Y SU PAPEL EN LA CREACIÓN DE COMUNIDADES DE APRENDIZAJE Y PARTICIPACIÓN
}

\author{
(THE ROLE OF MOOCS IN THE CREATION OF LEARNING AND PARTICIPATION COMMUNITIES)
}

Daniel Torres Mancera

David Gago Saldaña

Fundación Centro Superior para la Enseñanza Virtual, CSEV (España)

\section{RESUMEN}

Pese a su creciente popularidad y protagonismo, el valor más prometedor de los MOOC no deriva de lo que son, sino de lo que pueden llegar a ser, es decir, de las derivadas positivas que están empezando a aflorar y que tienen mucho que ver con el carácter flexible y abierto del aprendizaje que preconizan (Yuan y Powell, 2013). Estas posibilidades dimanan de algunos de sus rasgos, como son su modularidad, escalabilidad y capacidad de recombinación. Todo ello permite transitar hacia experiencias de aprendizaje personalizadas, en donde las personas pueden seleccionar sendas de aprendizaje ajustadas a sus preferencias y competencias.

Esta es la lógica subyacente al concepto de comunidades de aprendizaje y participación propuesta en este artículo postulado por CSEV, y que se ha hecho tangible a partir de la creación de una nueva plataforma "paraguas" que contiene las ya existentes (como UnX) y otras nuevas que se están incorporando.

Palabras clave: MOOCs, comunidades de aprendizaje y participación, aprendizaje personalizado, conectividad.

\begin{abstract}
Despite its increasing popularity, the most promising value of MOOCs is not derived from what they are, but from what they may transform into, that is, the positive derivatives that have already started to flourish and are very much related to the open and flexible character of learning advocated by MOOCs (Yuan and Powell, 2013). These capabilities arise from its very nature, namely the modularity, scalability and (re) combinative nature, thus enabling the transition towards personalised learning pathways that are also more adjusted to individual competences and preferences.
\end{abstract}

This is the rationale underlying the concept of learning and participation communities highlighted in this article by CSEV and underpinned by the creation of a new "umbrella" 
platform that brings together initiatives already implemented (such as UnX) and new ones that are now being incorporated.

Keywords: MOOCs, communities of participation and learning, personalised learning, connectivity.

\section{CONTEXTO: MOOCS Y DISRUPCIÓN}

Un proceso de cambio sin precedentes está aconteciendo en la educación en estos primeros años del siglo XXI. Así lo atestiguó el propio Rector del MIT, Rafael Reif, quien en su conferencia inaugural del año pasado, declaró que “(...) los nuevos modelos del e-Learning y el desarrollo de la tecnología están haciendo transitar hacia la educación superior hacia la mayor transformación tecnológica en los últimos 500 años". Es tal la profundidad de estos cambios que para muchas voces estamos asistiendo a una auténtica reinvención de los fundamentos del sistema, catalizada por las extraordinarias posibilidades ofrecidas por el desarrollo de las Tecnologías de la Información y las Comunicaciones (TIC).

Esta nueva etapa tiene al desarrollo de los MOOCs como una de sus manifestaciones más evidentes y de mayor recorrido; de hecho, para algunos autores (a modo de ejemplo, Conole, 2013), representan el último ejemplo en la línea de las tecnologías disruptivas descritas por Christensen (1997) ${ }^{1}$. Precisamente la educación es una de las actividades más proclives a experimentar estos procesos de innovación disruptiva, dado el tardío impacto que la tecnología está teniendo sobre ella (Regalado, 2012).

Pese a su carácter reciente (el primer experimento MOOC data del año 2008, aunque es cierto que hunde sus raíces mucho antes), los MOOCs se convirtieron en los protagonistas absolutos de la educación en el año 2012 (Daniel, 2012). Su reciente popularidad se explica a partir del momento en el que algunas de las universidades más prestigiosas de Estados Unidos acogieron con entusiasmo el concepto a través de iniciativas como EdX, Coursera o Udacity. Precisamente es el desarrollo de estas plataformas el que permite hablar de dos grandes categorías de MOOCs. Por un lado, los cMOOCs, derivados del enfoque conectivista de los precursores Siemens y Downes se apoyan en un aprendizaje auto-organizado, centrado en obtener el significado de la experiencia de aprendizaje con la comunidad, para lo cual resultan claves las herramientas de participación como los blogs, feeds, RSS y otros métodos descentralizados. Por su parte, los xMOOCs, ejemplificados por estas iniciativas nacidas en el mundo anglosajón, hacen hincapié en el dominio del contenido; los 
cursos se centralizan en un único sitio web y utilizan las herramientas de clasificación automatizada para soportar la actividad de cientos de miles de estudiantes.

En todo caso, debe enfatizarse que esta clasificación entre xMOOC y cMOOC ha quedado ampliamente superada, y de hecho la literatura especializada está aportando nuevas taxonomías que recogen su alto componente de cambio y dinamismo. Así, por ejemplo, Clark (2013), distingue entre ocho tipos de MOOC:

- transferMOOC: cursos ya disponibles que son trasladados a formato MOOC;

- madeMOOCs: más innovadores, hacen uso efectivo del vídeo y de material interactivo y tienen un nivel de calidad superior;

- $\quad$ synchMOOCs: tienen una fecha fija de comienzo y de finalización;

- asynchMOOCs: no hay fecha fija de comienzo ni de finalización, hay flexibilidad en los plazos de entrega;

- adaptiveMOOCs: proporciona experiencias de aprendizaje personalizadas, basadas en evaluaciones dinámicas y el uso de una gran cantidad de datos recogidos en el curso (learning analytics);

- groupMOOCs: el foco se encuentra en la colaboración que acontece entre pequeños grupos;

- connectivistMOOCs: énfasis en la conexión entre una red de participantes;

- miniMOOCSs: mucho más limitados en tamaño con respecto al concepto tradicional.

Otra clasificación es la de Conole (2013), propuesta a partir de doce dimensiones: grado de apertura, escala de participación (masificación), uso de multimedia, uso de comunicación, alcance de la colaboración con otros participantes, tipo de aprendizaje (centrado en las necesidades del estudiante, o, por el contrario, más centrado en el docente y más estructurado), nivel de aseguramiento de la calidad, fomento de los procesos auto-reflexivos, nivel de evaluación, carácter formal/informal, autonomía y diversidad.

Pese a todo, el valor más prometedor de los MOOC no deriva de lo que son, sino de lo que pueden llegar a ser, es decir, de la toma en consideración de todas las derivadas positivas (externalidades, en la jerga de los economistas), que ya están 
empezando a aflorar. De hecho, las taxonomías que empiezan a aparecer atestiguan el carácter cambiante y evolutivo de los MOOC, hasta tal punto que, para algunos autores, no terminarán llamándose así, y lo que es, más importante, tendrán poco que ver tal y como lo conocemos actualmente (Zapata-Ros, 2013).

Estas posibilidades se derivan de algunos de sus rasgos más singulares, como son su modularidad, escalabilidad y capacidad de recombinación. Todo ello permitirá transitar hacia experiencias de aprendizaje personalizadas y adaptadas, en donde las personas podrán seleccionar sendas de aprendizaje que se ajusten a sus preferencias y competencias. De hecho, los MOOCs promueven un cambio radical en la metodología de aprendizaje y enseñanza, rompiendo el esquema lineal que ha predominado tradicionalmente y promoviendo una implicación más evidente del estudiante en estos procesos, que puede elegir la dirección en la que explora el contenido de aprendizaje (Mackness et al., 2010). Adicionalmente, los participantes en un MOOC se convierten en miembros de la comunidad que aportan su conocimiento para que otros aprendan, participando entonces en un proceso de co-creación del que sus herramientas devienen sus canales de transmisión. De hecho, esta interacción se percibe como muy relevante por los propios participantes en estos contextos (Khalil y Ebner, 2013).

Asimismo, estas experiencias de aprendizaje pueden, además, transformarse en oportunidades en otros contextos económicos y sociales. Los resultados más relevantes de los MOOC son los relacionados con su capacidad para conectar mundos que habían permanecidos distantes hasta el momento. De hecho, los MOOC pueden favorecer la creación de nuevas formas de participación cívica y gobierno abierto, donde el conocimiento compartido y el intercambio de ideas se utilice no sólo para identificar el talento sino, lo que es más importante, para extraer propuestas útiles que ayuden a modelar los procesos de toma de decisiones en los ámbitos empresariales y de políticas públicas. Estas formas pioneras de participación, con grupos de interés común, bien arraigados y conectados globalmente, han llegado con los MOOC y van a revolucionar nuestra forma de construir Sociedad.

Esta es la lógica subyacente al concepto de comunidades de práctica y aprendizaje por la que aboga CSEV y otros socios. Construyendo a partir de UnX, la primera comunidad iberoamericana de emprendimiento digital, se ha creado una nueva plataforma "paraguas" para experimentar e implementar este concepto. Esto significa que UnX interactuará con otras iniciativas sectoriales (algunas ya existentes y otras novedosas) para promover nuevas y más ricas experiencias de participación y aprendizaje. 


\section{LOS COMIENZOS: UnX}

\section{OBJETIVOS Y JUSTIFICACIÓN DE UnX}

UnX es una innovadora comunidad online de emprendimiento que promueve la colaboración y la educación abierta en España, Portugal y Latinoamérica, nacida para el cumplimiento de los siguientes objetivos:

- Promover el aprendizaje permanente online, particularmente de las competencias y habilidades necesarias en la economía digital, como competencias digitales, desarrollo de aplicaciones móviles, idiomas y nuevos modelos de negocio. A través de esta estrategia de formación permanente online se puede capacitar y acreditar capacidades y competencias no reconocidas ni certificables actualmente, pero de máximo interés en la actualidad;

- Fomentar el emprendimiento basado en el conocimiento;

- Promover la inclusión en la educación superior, centrándose especialmente en los estudiantes, los desempleados y los subempleados.

El elemento aglutinador de UnX es su evidente vocación innovadora, que puede aproximarse desde una triple perspectiva:

- Innovación Tecnológica: fomento de la innovación y la generación de nuevos recursos tecnológicos aplicables a la educación virtual como herramienta para facilitar el acceso a la capacitación profesional y la formación superior del futuro.

- Innovación Metodológica: estudio y experimentación de nuevas metodologías y estrategias pedagógicas disruptivas apoyadas en el uso de la tecnología e internet (innovación funcional de plataformas de e-Learning, aprendizaje entre pares, aprendizaje social, aprendizaje autodidacta, aprendizaje masivo...).

- Innovación Abierta en recursos educativos y procesos: exploración de posibilidades y pilotaje de iniciativas innovadoras en el uso de repositorios abiertos de contenidos educativos, nuevas formas de acreditación y certificación abiertas, utilización de recursos virtuales en estas plataformas y creación de una comunidad de desarrollo abierta.

El proyecto UnX es el resultado del esfuerzo conjunto inicial realizado por el Centro Superior para la Enseñanza Virtual (CSEV), Telefónica, Santander, la 
Universidad Nacional de Educación a Distancia (UNED), y el Center for Mobile Learning del MIT (Massachusetts Institute of Technology). En cualquier caso, la naturaleza abierta de la Comunidad de Emprendimiento UnX deja abierta la puerta para aquellas entidades, que deseen involucrarse en la iniciativa. Estos son los casos, por ejemplo, de RedEmprendia (red de universidades que promueve la innovación y el emprendimiento responsable en Iberoamérica) o de CEDERJ (Centro Universitario de Educación a Distancia del estado de Río de Janeiro, encargados de impulsar UnX en lengua portuguesa), que se han unido recientemente al proyecto.

\section{FUNDAMENTOS METODOLÓGICOS}

UnX se basa en la impartición de MOOC. Los MOOC se nutren de contenidos abiertos y flexibles de páginas web, wikis, recursos educativos abiertos y redes sociales, y pueden escalarse para ajustarse a la demanda. Es un aprendizaje en el que los conocimientos se adquieren de la comunidad y de los sujetos que trabajan juntos (aprendizaje social y peer to peer).

Las actividades propuestas siguen una metodología basada en retos y juegos, con objeto de que los participantes puedan participar de forma práctica y lúdica como parte motivacional del aprendizaje. En este contexto, el papel lúdico que nos permite incorporar la gamificación va a jugar un rol fundamental. El concepto de gamificación tal y como aquí se plantea se refiere al uso de la lógica y mecanismos provenientes del mundo de los juegos en la creación de contenidos y metodologías educativas. La utilización de las técnicas de juego en ámbitos no lúdicos no implica una novedad en sí, pero su combinación con las posibilidades ofrecidas por las TIC y, en este caso con los MOOCs, abre un nuevo, enorme y apasionante abanico de posibilidades para los educadores. No obstante, el proceso de incorporar la gamificación a la experiencia educativa requiere de un esfuerzo que permita adaptar sus potencialidades a la realidad de la educación. La creatividad es, por tanto, una cualidad de suma importancia a la hora de llevar a cabo una integración efectiva y eficiente de estos conceptos. Compromiso, diversión, motivación, curiosidad, inmersión, colaboración, creatividad constituyen características que forman parte del uso de los juegos que, sin duda alguna, suponen un desafío y una necesidad sumamente relevantes para incorporar a los MOOCs.

UnX utiliza un sistema de acreditación y certificación online basado en la consecución de insignias o badges, así como un modelo de certificación oficial que ya está dando sus primeros pasos. 
En cuanto a las insignias o badges son indicaciones visuales de niveles de rendimiento, capacidades o conocimientos que avalan que una persona ha seguido de manera activa una lectura, un debate, un grupo de trabajo o un evento de asistencia virtual.

Respecto a la certificación oficial, el pasado mes de julio se emitió el primer certificado en UnX, extendiéndose durante el mes de noviembre a todos los cursos esa funcionalidad, previo pago de unas tasas simbólicas. El certificado será propio de la plataforma, esto es, certifica UnX, no las Universidades u organismos vinculados a UnX, si bien éstas pueden, si lo estiman conveniente, generar mecanismos de convalidación de esos cursos con créditos ECTS.

Además, con el objetivo de ayudar a la auto-organización de los usuarios en una comunidad masiva, se implementó un sistema de karma, que acredita la actividad de sus participantes. Este karma o indicador de reputación social es el indicador de rendimiento de la participación de todos los usuarios en la comunidad a través del foro, preguntas y respuestas y el blog. Sobre la base de un sistema de puntuación, se medirá el impacto en el rendimiento del usuario y su relevancia en la comunidad, dando derecho a los usuarios a tener acceso a características adicionales. Este karma tiene un componente dinámico, es decir, que la puntuación puede bajar si la participación descendiera a lo largo del curso. La consecución de ciertos valores de karma puede conllevar la adquisición de un grado permanente de perfil de usuarios avanzados (dinamizadores). Estas recompensas mejoran la motivación y la participación en los canales sociales y supone una menor dependencia en los profesores del curso, lo que resulta especialmente importante si tenemos en cuenta que puede haber miles de estudiantes registrados en un MOOC.

Otro de los aspectos claves relacionado con el desarrollo de UnX es el relativo a la evaluación de las actividades y evolución de los estudiantes. A este respecto las actividades responden fundamentalmente a dos métodos: autoevaluación y evaluación entre pares. De forma complementaria, se considera necesario implementar en ciertos casos otro método que permita llevar a cabo el proceso evaluador. En el caso de algunos ejercicios y prácticas complejas, es necesario disponer de procesos de evaluación y validación supervisados por personas.

La autoevaluación o self-assessment se articula mediante diferentes tipos de cuestionarios o quizzes, ejercicios de respuesta única $\mathrm{u}$ otras actividades autocorregibles. 
Por su parte, la evaluación entre pares o peer review requiere un mayor esfuerzo en la definición de los criterios de evaluación y las métricas a utilizar. Está basada en la interacción con la comunidad, el valor percibido por ésta en relación a las aportaciones de los usuarios y la valoración de prácticas y ejercicios por parte de otros iguales (peers).

La evaluación y validación tutorizada es el mecanismo de mayor control, pero también el que demanda más dedicación de recursos. Este tipo de evaluación se articula mediante la evaluación por parte de curators, expertos o profesores de ejercicios o prácticas complejas, que requieran la supervisión de personas para la comprensión crítica de los resultados de la tarea.

No obstante, lo que desde el principio ha hecho realmente diferente a UnX del resto de plataformas de MOOC conocidas es su sentido de comunidad (Santamaría, et al., 2013). De hecho, UnX no sólo combina cursos que promueven el emprendimiento y el autoempleo con las redes sociales, sino que además trabaja con empresas para publicar noticias e información sobre eventos y oportunidades de trabajo tanto online como offline. Los nuevos emprendedores tienen acceso a tutorización y mentorización, información financiera, business angels, becas y otras oportunidades. En este sentido, UnX funciona como un hervidero donde se satisfacen diferentes necesidades y demandas, ofreciéndose capacidades y oportunidades laborales.

\section{CURSOS DE UnX}

En la actualidad son cinco los MOOCs disponibles en la plataforma UnX, aunque como se precisará más adelante, ese número va a crecer sensiblemente en breve:

- Emprendimiento y Desarrollo de Aplicaciones Móviles con App Inventor. Este curso busca la creación de una comunidad iberoamericana de desarrolladores y emprendedores en App Inventor, la plataforma abierta del Instituto Tecnológico de Massachusetts (MIT) para la creación de aplicaciones Android, creada por Google y que actualmente lidera el propio MIT para su difusión. Con la puesta en marcha de un MOOC en la herramienta creada por el MIT, cualquier persona que se inscriba, aprenderá a programar aplicaciones para móviles y recibirá las nociones básicas para llevar a cabo el proceso de comercialización de las mismas de una forma sencilla.

- Competencias Transversales del Emprendedor de la Comunidad UnX, centrado en potenciar los valores del espíritu emprendedor y en apoyar las iniciativas de creación de nuevas ideas de negocio. 
- Capacidades Digitales Básicas, liderado conjuntamente por la UAPA(Universidad Abierta para Adultos de República Dominicana) y la UNED. Este curso se integra dentro de la línea de Capacitación de IBERVIRTUAL ${ }^{2}$, y su objetivo reside en impulsar la capacitación en competencias digitales básicas, intentando reducir la posible brecha digital, dotando a los alumnos de los conocimientos básicos necesarios para desenvolverse en la realidad digital del siglo XXI.

- Mitología para Emprendedores. Se trata de que el emprendedor conozca claves fundamentales que le ayudarán a asegurar el éxito de su plan empresarial, atendiendo a modelos míticos que la cultura occidental ha adoptado como paradigmáticos y a las enseñanzas de ellos derivadas. Es un MOOC ofrecido por el Departamento de Filología de la Universidad de Alcalá.

- Inglés en mil Palabras. Se parte de la extraordinaria importancia que tiene desenvolverse en inglés como lenguaje de los negocios y del potencial comunicativo de las mil palabras más usadas porque éstas constituyen más del 70\% de la expresión escrita y oral en inglés todo lo que se dice y escribe en inglés. Se trata de un MOOC ofrecido por el Departamento de Filologías Extranjeras y sus Lingüísticas de la UNED.

En cuanto a los cursos en cartera para el futuro inmediato, hay un total de cinco, muy diversos en temáticas pero unidos dentro del marco común de fomento de la capacidad emprendedora:

- Mini-curso Desarrollo STEMx Realidad Aumentada Aplicaciones Móviles;

- Mini-curso Design de Aplicativos Móveis STEMx --Sem necessidade de programação (portugués);

- Mini-curso Diseño de Aplicaciones Móviles STEMx --Sin Necesidad de Programación;

- Emprendimiento e innovación social;

- Aplicativos móveis e empreendimento com o App Inventor (portugués).

\section{UnX SE HACE UBICUO: "LEARNING ON THE GO"}

UnX es consciente de la necesidad de apostar por un aprendizaje ubicuo, flexible y accesible, y el carácter clave que dentro de este escenario aporta nuevas soluciones 
de movilidad. Esto es especialmente relevante dada la fuerte presión que existe en términos de demanda, y que tiene formación a lo largo de la vida a uno de sus grandes motores.

Con objeto de transitar hacia un escenario de aprendizaje completamente ubicuo, y como iniciativa novedosa, UnX se está haciendo móvil. En este sentido, "Learning on the go" (Aprender sobre la marcha) es la plataforma móvil diseñada específicamente para fomentar el aprendizaje, el desarrollo profesional y el emprendimiento para todos.

"Learning on the go" proporciona experiencias educativas de primera clase (nunca inferiores) que:

- Se adecuan a la disponibilidad y el estilo de vida de cada persona (aprender en el autobús, en el tren, en casa, etc.);

- Añaden valor y complementan otras iniciativas educativas, tanto presenciales como online;

- Conectan a los estudiantes con redes, contenidos y oportunidades relevantes personal y localmente;

- Permite a los empresarios en ciernes probar nuevas ideas e iniciativas, aportando su visión a la experiencia de aprendizaje y viceversa.

Las principales características y funcionalidades de la plataforma se pueden sintetizar en las siguientes ideas:

- Adaptación al horario y ubicación del usuario y a las peculiaridades del dispositivo móvil (tamaño de pantalla, resolución, etc.);

- Acceso al contenido sin conexión, incluso con dispositivos de gama media;

- Disponibilidad en varios idiomas;

- Calendario de eventos y oportunidades locales;

- Contacto en redes sociales con compañeros y mentores;

- Mecanismos de certificación; 
- Analíticas de uso para nuevas oportunidades de negocio / campañas;

- Otras prestaciones para dispositivos móviles, como canjeo de créditos de clase por crédito para el móvil y otros mecanismos de motivación; funciones de localización; realidad aumentada, uso de SMS, voz y otros canales de comunicación.

\section{UnX: ALGUNOS HITOS HASTA LA FECHA}

En sus primeros diez meses de vida (septiembre 2013), UnX ha registrado más de 21.000 miembros, de los cuales $\mathbf{1 5 . 5 0 0 ~ ( 7 3 \% ) ~ e s t a ́ n ~ a p u n t a d o s ~ a ~ c u r s o s ~ y ~} 4.700$ (27\%) simplemente participan en las actividades de la comunidad.

En este sentido, la consolidación de UnX desde la perspectiva del número de registrados es un hecho incontestable. Tal y como se aprecia en el gráfico 1, la evolución del número de registros desde marzo a septiembre de 2013 ha sido muy notable, ya que se ha multiplicado por cuatro. A ello ha contribuido tanto el incremento de los registros en cursos ya disponibles desde el comienzo (caso del curso de Emprendimiento y desarrollo de aplicaciones móviles con App Inventor), como la incorporación de nuevos cursos al portfolio.

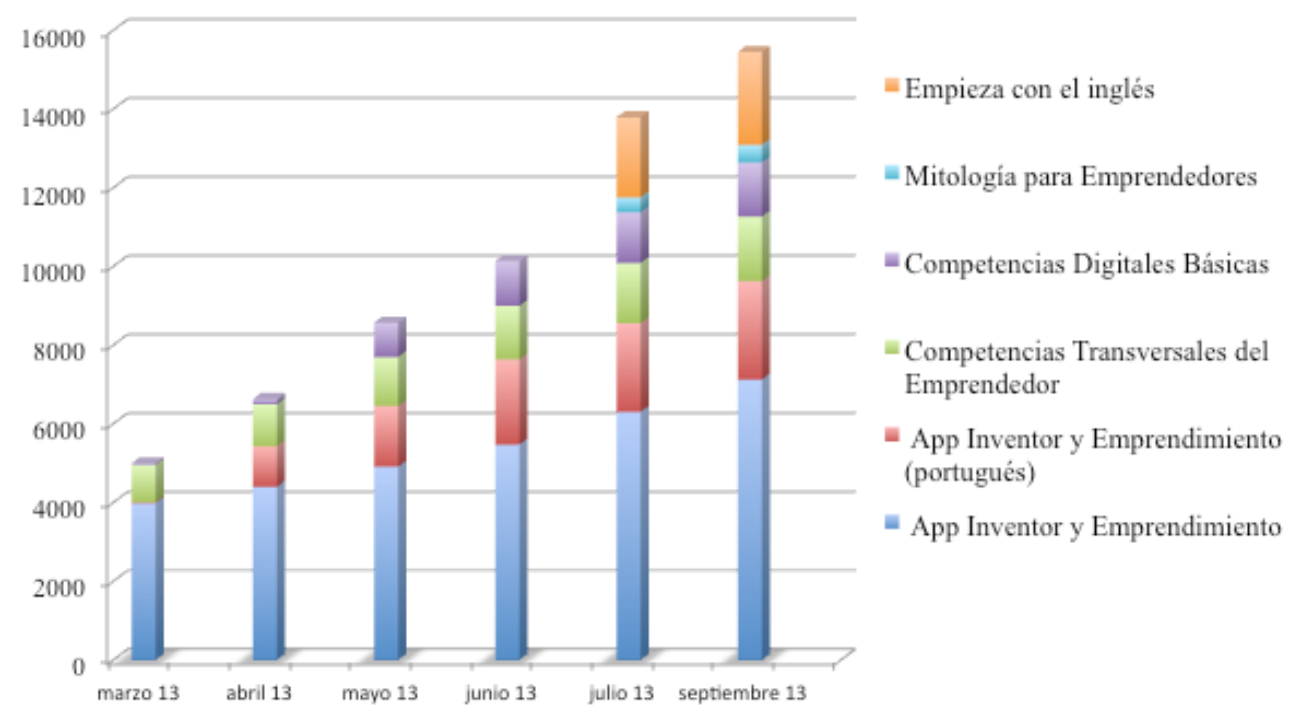

Gráfico 1. Registrados en cursos UnX: evolución marzo-septiembre 2013. Fuente: UnX3 
Por su parte, como se puede apreciar en el gráfico 2, UnX es una iniciativa global en el ámbito latinoamericano. Es cierto que algo más de la mitad de las visitas proceden de España, pero hay una representatividad muy significativa de países latinoamericanos, que además crece en la medida que UnX se consolida. En todo caso, son más de 80 nacionalidades distintas las representadas dentro de UnX.

Muchas universidades e instituciones europeas, americanas e iberoamericanas están trabajando con UnX para ofrecer formación empresarial y oportunidades de trabajo en la economía digital. Como se acaba de citar, además de la UNED y el MIT, la Universidad de Alcalá y la UAPA (Universidad Abierta para Adultos de la República Dominicana) están ofreciendo MOOC en UnX, mientras que otras instituciones mediante iniciativas como HP Catalyst, Qualcomm Wireless Reach y Escuela Virtual Mercosur están patrocinando cursos y retos. Por último, otras organizaciones participan en la financiación, liderando proyectos, lanzando plataformas tecnológicas o creando contenidos para nuevos MOOC.

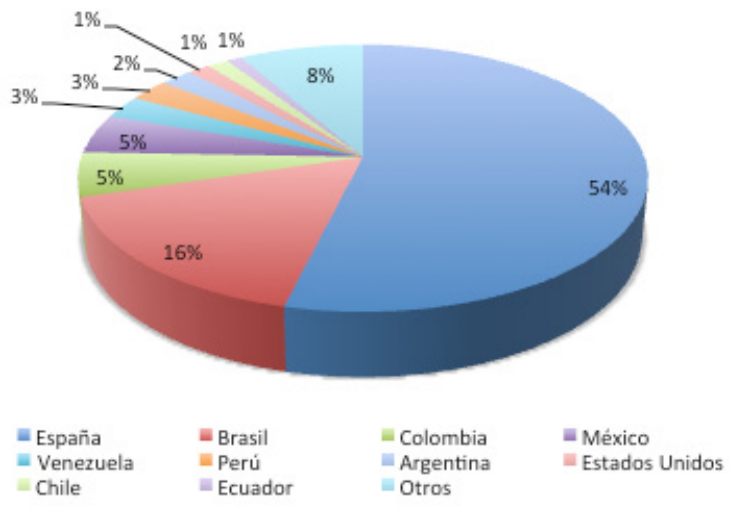

Gráfico 2. Procedencia geográfica de las visitas a UnX (septiembre 2013). Fuente: UnX

Por todo ello, CSEV fue merecedor del Premio Centro de Excelencia 2013 del New Media Consortium (NMC), que reconoce la excelencia demostrada y los grandes logros en la aplicación de tecnologías a la enseñanza, el aprendizaje y la investigación creativa, por el desarrollo de los entornos de aprendizaje online de UnX.

\section{LA CONTINUACIÓN: WEPRENDO}

El pasado mes de julio, CSEV y algunos de sus socios lanzaron una nueva comunidad online abierta y masiva para el desarrollo de aplicaciones y el emprendimiento. Esta plataforma educativa proporciona acceso a MOOCs para la 
creación de aplicaciones móviles utilizando tecnología punta de Qualcomm y permite a los estudiantes formar parte de una comunidad de emprendimiento móvil global donde pueden compartir experiencias y conocimiento. El objetivo del proyecto es fomentar una fuerza de trabajo capacitada para las necesidades impuestas por la nueva economía digital e impulsar la empleabilidad y el emprendimiento de los jóvenes desempleados en España e Iberoamérica.

Este MOOC se utilizará para enseñar tecnologías Qualcomm, como la tecnología de realidad aumentada Vuforia ${ }^{\mathrm{TM}}$, la de comunicación P2P Alljoyn ${ }^{\mathrm{TM}}$ y la plataforma sensible al contexto Gimbal ${ }^{\mathrm{TM}}$ para Android e iOS. La plataforma educativa online alberga materiales educativos como lecciones, casos de estudio, vídeos, retos y herramientas interactivas. Los estudiantes utilizan dispositivos móviles inteligentes como tabletas para acceder a los cursos, aprender de profesores de universidad y empresarios de éxito y conectarse con la comunidad online. Es más, algunos cursos utilizarán un enfoque semipresencial combinando MOOC con talleres y seminarios presenciales específicos para experimentar con Vuforia ${ }^{\mathrm{TM}}$ y Alljoyn ${ }^{\mathrm{TM}}$.

\section{(9) weprendo}

Ilustración 1. Logo Weprendo

En la actualidad hay un MOOC disponible en Weprendo, Empredimiento y Desarrollo de Aplicaciones de Realidad Aumentada, que tiene por objeto introducir a los nuevos emprendedores en el campo de la Realidad Aumentada para dispositivos móviles y su aplicación en diferentes campos profesionales haciendo uso del SDK (software development kit) de Vuforia sobre Unity3D, una herramienta visual para crear apps/juegos multiplataforma. La metodología de aprendizaje es similar a la de UnX, por cuanto se basa en retos que definen una serie de módulos a cuya finalización se obtiene un badge, siempre que se supere una evaluación que tiene un componente de autoevaluación y otro entre pares.

Además, de forma inminente se van a lanzar dos nuevos cursos relacionados con el desarrollo de apps móviles sobre Android (Alljoyn SDK y Gimbal SDK). 


\section{CREANDO UNA COMUNIDAD DE COMUNIDADES: COLMENIA}

La transición desde el concepto tradicional de MOOC a una visión más amplia del mismo como herramienta para la participación online requiere de la arquitectura apropiada desde el punto de vista tecnológico. La lógica subyacente a esta plataforma «paraguas» que se está creando es, no sólo que sirva como «contenedor» último de todos los contenidos, sino que también, y lo que es más importante, proporcione el «pegamento» necesario para conseguir la interacción y las sinergias entre los diferentes componentes. El verdadero valor de la comunidad, que tendrá por nombre Colmenia, es, por tanto doble; por una parte, es una plataforma para ensayar e implementar contenidos (y pedagogías asociadas) novedosos: así, más allá de los MOOC contemplados en su concepción más tradicional, se pondrán en práctica experiencias blended ligadas a los mismos, o incluso mOOCs (micro open online courses), que cambian el componente masivo por el de la absoluta autonomía en la creación de las experiencias por parte de sus miembros, por cuanto cualquiera puede poner al servicio de la misma su experiencia, competencias y conocimiento en forma de curso abierto y online, enriqueciéndose notablemente el componente de aprendizaje social (Marquis, 2013). Las comunidades pueden no ser sólo de aprendizaje, sino también de carácter práctico, donde, como ya se está haciendo ya en UnX o WePrendo, los participantes interactúan para crear y emprender juntos, fomentándose de esta forma el acervo de habilidades blandas (soft skills), que asumen un papel tan crítico dentro de las denominadas "habilidades y cualificaciones del siglo XXI" (Trilling y Fadell, 2009).

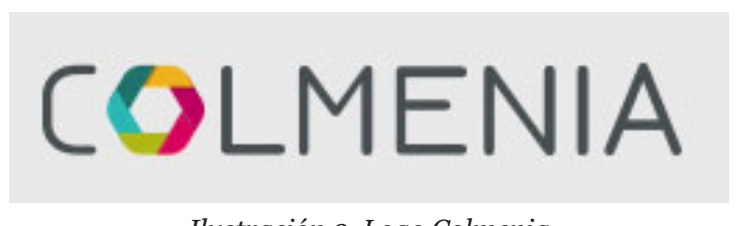

Ilustración 2. Logo Colmenia

Por otro lado, Colmenia tiene un valor sinergético por cuanto amplifica las capacidades de cada uno de sus elementos, fundamentalmente porque las posibilidades de recombinación a que dan lugar pueden alumbrar nuevas y más ricas realidades. En este sentido, sirve también para optimizar una de las características diferenciales de los MOOC con respecto a otras fórmulas de aprendizaje, como es la explotación del "long tail", que permite acoger dentro de la comunidad cualquier sensibilidad o interés por minoritario que éste sea (McAuley et al., 2010). 
Con todo ello, Colmenia va a suponer, en última instancia:

- La creación de una comunidad de comunidades sumamente centrada en la experiencia del usuario. De hecho, una de las lagunas que se observa en la formación online en la actualidad es que se encuentra muy fragmentada e institucionalizada, de tal modo que el usuario puede experimentar dificultades que deriven en deficiencias educativas o, lo que es peor, altas tasas de abandono ${ }^{4}$. Se han introducido diversas medidas para atajar estos cuellos de botella. Por ejemplo, será necesario identificarse una sola vez en la comunidad, independientemente de la plataforma (UnX, Weprendo u otra). Además, el estudiante podrá controlar totalmente su entorno personal de aprendizaje de diferentes maneras:

- Acceso a un apartado personal (marcapáginas/agenda y contactos/ mi actividad/mis cursos y rendimiento (indicadores de analítica del aprendizaje)/recomendaciones/badges\&certificación/).

- Acceso al área educativa (cursos ofrecidos/tests de nivel/gestión de clases virtual).

- Acceso a datos de emprendimiento/comunidad (mediateca/noticias/ blog/asistencia a eventos utilizando herramientas de geolocalización/ mentorización/participación en talleres y conferencias virtuales/portafolio de proyectos/convocatoria de propuestas).

- Proporcionar a los estudiantes las herramientas necesarias para «navegar» por el espacio educativo online, fomentar la reflexión sobre sus procesos de aprendizaje y siguientes pasos.

- Diferentes tipos de tecnologías interconectadas de diversos modos y oportunidad de crear nuevos proyectos en el futuro.

- Consecución de super badges. El concepto de super badge está muy alineado con la implementación de entornos personales de aprendizaje en el contexto de los MOOC. Un super badge es el resultado de unir diferentes badges que pueden conseguirse por finalizar un MOOC, participar en debates abiertos y foros, concursos, asistir a eventos educativos presenciales, etc. Esto significa que los badges pueden conseguirse de diferentes plataformas y combinarse libremente, siguiendo diferentes sendas de aprendizaje. Dichas sendas son sugeridas por la propia plataforma y para que sean óptimos el usuario ha de definir tanto sus 
competencias y formación actual, como sus preferencias para la adquisición de nuevas competencias o habilidades.

Como se observa en la ilustración 3, Colmenia va a aglutinar comunidades sectoriales de muy distinta índole. Ámbitos como la tecnología y el emprendimiento, cultura, género, responsabilidad social corporativa, salud y educación deben tener su espacio, sin prejuicio de que surjan otras en la medida que se vaya detectando la necesidad y el interés.

GLOOC: Global - Local Online Open Communities

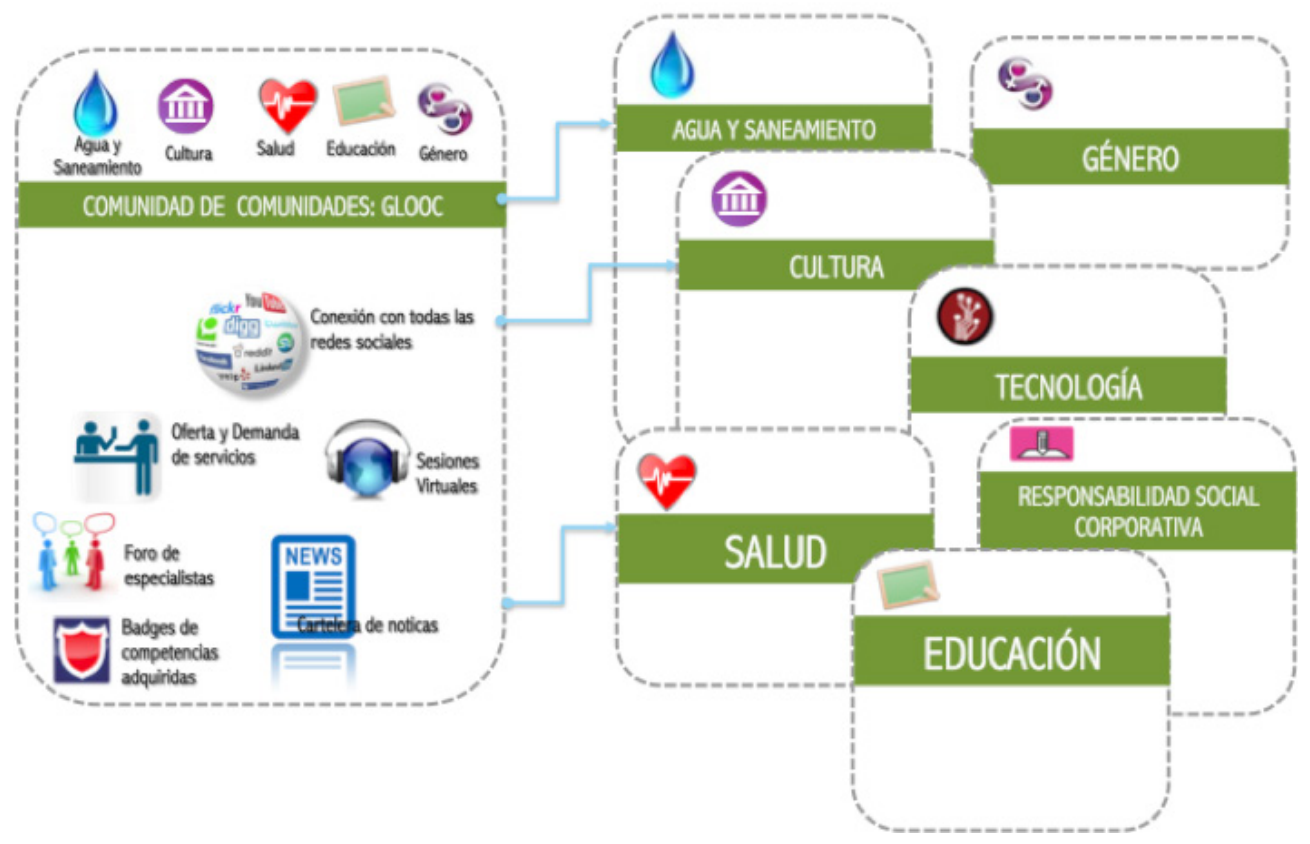

Ilustración 3. Una comunidad de comunidades. Fuente: CSEV

Con todo ello, Colmenia constituirá un exponente de una nueva forma de aprender, basada en promocionar la adquisición de un mayor número de competencias al máximo número de participantes, en contraposición de un modelo que se antoja caduco y que se basa en promocionar conocimientos a un número limitado de estudiantes y con resultados muchas veces inciertos (Roberts y Greteman, 2013).

Conviene apuntar que la iniciativa va a suponer poder ensayar sobre el terreno algunos principios conceptuales relativos al aprendizaje colaborativo y en comunidad. Pese a que surgió mucho antes de que aparecieran los MOOCs, el trabajo 
de Bruner (1996) se adentra de lleno en el análisis del aprendizaje en comunidad abundando en el modelo de Brown (1994). Bruner define cuatro ámbitos de este tipo de aprendizaje: agencia, por el que las personas que aprenden toman el control de su propio proceso de aprendizaje. El segundo es la reflexión, que significa la apreciación que hacen de lo que aprenden, y que constituye un elemento clave del aprendizaje autoregulado (Zimmerman, 2000). El tercero es la colaboración, por el que los individuos trabajan conjuntamente en el entorno de aprendizaje, y el cuarto es la cultura, esto es, la forma en que construimos, negociamos y cristalizamos y hacemos "real" ese aprendizaje. Como se puede observar, los cuatro ámbitos están de lleno presentes en Colmenia. En primer lugar, la agencia supone que los entornos de aprendizaje incentivan a los participantes a elegir para dar cobertura a sus necesidades de formación; la conformación de los entornos de aprendizaje personalizados en la plataforma permite al participante ser autónomo en este aspecto y tomar sus propias decisiones. Por otro lado, la reflexión está presente no sólo en la autoregulación del aprendizaje que consiguen los participantes (que se beneficia en gran medida de la interacción con otros), sino también a la disponibilidad de herramientas que tiene la plataforma para que el participante haga consideraciones, refuerce lo aprendido y se reconozcan sus hitos de aprendizaje. Poco hay que decir de la colaboración, convertido en el eje del proceso de aprendizaje, mientras que la cultura ofrece un campo de experimentación en esta iniciativa muy sugerente. En ese aspecto, convendría preguntarse ¿̇surgirá una sola cultura, la cultura de la comunidad de comunidades o, por el contrario, habrá matices dependiendo de la comunidad?

Las dinámicas de aprendizaje colaborativo alumbradas por esta apuesta podrán igualmente extenderse hacia esquemas de participación masiva más general, fomentando nuevas formas de e-participación. Yendo aun más lejos, pueden generarse dinámicas de aprendizaje que, al mismo tiempo, sean esquemas de participación masiva. A modo de ejemplo, se pueden crear cursos, por ejemplo, en el área de la economía ("nuevos modelos de productividad a corto y largo plazo", "perfiles profesionales imprescindibles en la economía digital”, podrían ser algunas alternativas), en los que las aportaciones y el conocimiento compartido por los participantes sirvan para identificar talento e incluso, en última instancia, extraer propuestas útiles en la toma de decisiones empresariales o la adopción de políticas públicas.

Este tipo de iniciativas conectan con el concepto de comunidades colaborativas de Adler y Hecksher (2006), basada en interacciones muy diversas en competencias y bases de conocimiento, y que además van cambiando a lo largo del tiempo. Se 
trata de comunidades (no necesariamente vinculadas al aprendizaje), de contornos más difusos, porosas, con mayor capacidad de conectarse y fusionarse con otras realidades, donde la experiencia y el saber hacer marcan una diferencia valorada por los otros miembros de la comunidad y que produce valores que se dirimen en el ámbito de la discusión pública, actuando éstos como factores orientadores.

Estos nuevos paradigmas de crowd-Citizenship y Crowd-Governance alimentados por la aparición de esta iniciativa van a suponer, desde luego, abrir nuevas formas de alfabetización digital hacia grandes capas de la sociedad y fomentar los mecanismos de interacción y colaboración en red, que están intrínsecos en la popularización de los MOOCs como concepto (Stewart, 2013). Asimismo, dan lugar a la emergencia de un nuevo concepto: GLOOP o, lo que es lo mismo, esquemas de GLocal - por Global y Local al mismo tiempo- Open Online Participation. Se trata de nuevas formas de participación en las que las comunidades se conectan globalmente gracias a Internet y tienen una fuerte implantación e impacto local, y en los que las cuestiones locales se perciben dentro un contexto global, de la misma forma que las cuestiones globales se perciben como resultado de las circunstancias locales. El carácter distribuido de la comunidad, que integra comunidades diferentes, es especialmente fructífero porque permite concitar dos tendencias que aparentemente pueden resultar antagónicas: por un lado, hace posible la especialización, y hacer cristalizar la participación en torno a grupos con intereses comunes. Pero, por otro, estas comunidades no son islotes aislados, sino que podrán interaccionar, activándose sinergias y puntos de encuentro entre ellas.

Estas nuevas formas de participación han llegado con los MOOC y comparten con ellos raíces y objetivos, y, además, están llamados a revolucionar nuestra forma de aprender, trabajar y construir Sociedad.

\section{CONCLUSIONES}

Los MOOCs constituyen una de las puntas de lanza de los cambios profundos que están aconteciendo en el ecosistema educativo. Algunos de sus rasgos (modularidad, escalabilidad y capacidad de recombinación) explican su carácter dinámico y su aptitud para reinventarse, ofreciendo posibilidades muy relevantes como herramienta no sólo de aprendizaje, sino también, en sentido más amplio, de participación e interacción.

Una de estas posibilidades es su integración dentro de entornos "paraguas", constituidos por comunidades en donde los participantes puedan diseñar sus propios entornos de aprendizaje de acuerdo con sus competencias y sus necesidades. Esas 
comunidades tendrán como uno de sus protagonistas a los MOOCs en su concepción más "tradicional" de uso exclusivo del canal online, pero también admitirán otras posibilidades, como los entornos blended o la creación de comunidades globales (online) y clubs locales (presenciales).

Estos entornos "paraguas", como Colmenia, dan cobertura a algunas de las tendencias novedosas por las que los MOOCs ya están transitando en la actualidad, pero, sobre todo, lo harán en el inmediato futuro. Una de estas tendencias es la ruptura del canal único (canal on line), pero hay otras, tales como: el alto potencial de los MOOCs en áreas específicas y “de nicho” (Ciencias Sociales, Escuelas de Negocio, etc.); la especialización hacia nuevas audiencias (como las personas desempleadas, personas que necesitan reciclar su formación, formación profesional y secundaria); la oferta de cursos más cortos, modulares, adaptados a las necesidades (frecuentemente muy particulares) o la creación de espacios flexibles de aprendizaje con agregación de herramientas sociales.

Adicionalmente, el concepto de comunidad que surge a partir de la plataforma paraguas tiene por detrás cierto andamiaje conceptual, que es incluso anterior a la irrupción de los MOOCs y de su contraparte epistemiológica, el conectivismo (Siemens, 2005). De hecho, el modelo FCL (fostering communities of learners) de Bruner (1996), enfatiza aspectos similares sobre todo con respecto al cómo y dónde se producen las experiencias de aprendizaje. Este modelo es útil, además, por su carácter holístico, ya que, como se acaba de comentar, permite introducir el concepto de multicanalidad en el aprendizaje (Irvine et al., 2013), en contraposición con en el enfoque conectivista, donde el canal es único (canal on line).

Por su parte, la multicanalidad, modularidad y conectividad de la iniciativa propuesta son rasgos que traen a un primer plano la visión transmedia aplicada a la educación. El concepto transmedia, propuesto por Henry Jenkins en un artículo publicado en Technology Review en el año 2003 con el objetivo de definir la técnica narrativa basada en la creación de mundos (narrativos) desarrollada a través de múltiples formas y plataformas, integrando experiencias (muchas de ellas de carácter interactivo), ya se ha instalado en el ámbito de la educación y el aprendizaje (Raybourn, 2012). El concepto de comunidad de comunidades descrito constituye, en este sentido, un extraordinario escenario para enriquecer el enfoque transmedia incorporando nuevas realidades educativas.

Por su parte, la extensión de la plataforma para dar cobertura a formas de participación más amplias abrirá la puerta a conceptos nuevos, como el GLOOP 
(global/local open online participation), que ayudarán en la consolidación de nuevas formas de cristalización de inteligencia colectiva y ciudadanía activa.

\section{AGRADECIMIENTOS}

Agradecemos el apoyo de los principales partners de UnX/Weprendo: UNED, CSEV, Telefónica, Banco Santander, Massachusetts Institute of Technology (MIT) y Qualcomm Technologies.

\section{NOTAS}

1. El propio Christensen también considera a los MOOCs como una transformación revolucionaria, tan importante como lo fue la popularización del motor gracias a Henry Ford (Horn y Christensen, 2013).

2. IberVirtual, aprobado en Mar del Plata (Argentina) en Diciembre de 2010, es un proyecto adscrito a las Cumbres Iberoamericanas de Jefes de Estado y de Gobierno. El principal objetivo de Ibervirtual es reforzar y potenciar la educación inclusiva mediante el fortalecimiento de la Educación a Distancia en el Espacio Iberoamericano del Conocimiento. De esta manera, con el desarrollo de Ibervirtual se pretende: fortalecer las Instituciones de Educación Superior que desarrollan Educación a Distancia; avanzar en nuevas modalidades de Educación a Distancia que permitan ofrecer más oportunidades de acceso a quienes se encuentran en situaciones de desigualdad; y mejorar la capacitación de los profesionales que se encargan de desarrollar la formación y la investigación en éste ámbito.

3. No hay medición correspondiente al mes de agosto 2013.

4. Aunque hay mucha divergencia por tipo de MOOC, la tasa de abandono se sitúa ampliamente por encima del 90\% de acuerdo con los estudios de Katy Jordan (ver, a tal efecto, Parr, 2013).

\section{REFERENCIAS BIBLIOGRÁFICAS}

Adler, P. S.; Heckscher, C. (2006). Towards a collaborative community. En: Heckscher, C.; Adler, P. S. (Eds.). The firm as a collaborative community: Reconstructing trust in the knowledge economy. Oxford: Oxford University Press, (11-105).

Brown, A. L. (1994). The advancement of learning, Educational Researcher, 23 (8), (4-12).
Bruner, J. (1996). The culture of education. Cambridge MA: Harvard University Press.

Conole, G. (2013). MOOCs as disruptive technologies: strategies for enhancing the learner experience and quality of MOOCs [documento en Google Docs], [en línea] Disponible en: https://docs. google.com/a/csev.org/document/d/1B 6QAx6OiwK3VW16idU7mnHDuZljyy6 
r7gLXhTzUa5co/edit?pli=1 [consultado 2013, 15 de septiembre].

Daniel, J. (2012). Making Sense of MOOCs: Musings in a Maze of Myth, Paradox and Possibility. [en línea] Disponible en: http://blog4222.blogspot.ca/2012/o9/ making-sense-of-moocs-musings-inmaze.html [consultado 2013, 14 de noviembre].

Horn M.; Christensen C. (2013). Beyond the Buzz, Where Are MOOCs Really Going? Opinión, Wired. [en línea] Disponible en: http://www.wired.com/ opinion/2013/02/beyond-the-moocbuzz-where-are-they-going-really/ [consultado 2013, 15 de septiembre].

Irvine, V.; Code, J.; Richards, L. (2013). Realigning Higher Education for the 21st Century Learner through Multi-Access Learning, MERLOT Journal of Online Learning and Teaching, 9 (2), (172-187).

Jenkins, H. (2003): Transmedia Storytelling, Technology Review, [en línea] Disponible en: http://www.technologyreview. com/news/401760/transmedia-storytelling/ [consultado 2013, 30 de septiembre].

Khalil, H.; Ebner, M. (2013). How satisfied are you with your MOOC? A Research Study on Interaction in Huge Online Course. Actas de AACE World Conference on Educational Multimedia, Hypermedia and Telecommunications, Victoria, (830-839).

Mackness, J.; Mak, S.; Williams, R. (2010). The ideals and reality of participating in a MOOC. Actas de Networked Learning Conference, Lancaster, (266-275).

Marquis, J. (2013). Resources \& Expectations For Creating A Smarter MOOC [blog TeachTought], [en línea] Disponible en: http://www.teachthought.com/ technology/resources-expectations-forcreating-a-smarter-mooc/ [consultado 2013, 25 de septiembre].

McAuley, A.; Stewart, B.; Siemens, G.; Cornier, D. (2010). The MOOC model for
Digital Practice, University of Prince Edward Island y Social Sciences and $\mathrm{Hu}-$ manities Research Council. [en línea] Disponible en: https://oerknowledgecloud.org/sites/oerknowledgecloud. org/files/MOOC Final o.pdf, Canadá [consultado 2013, 25 de septiembre].

Parr, C. (2013). Not Staying the Course [blog Inside Higher Ed]. [en línea] Disponible en: http://www.insidehighered. com/news/2013/05/10/new-study-lowmooc-completion-rates [consultado 2013, 30 de octubre].

Raybourn, E. M. (2012). Introduction to Transmedia for Training and Education, [Webinar impartido el 18 de diciembre de 2012]. [en línea] Disponible en: http://www.adlnet.gov/wp-content/ uploads/2013/01/RaybournTransmedia-Webinar-12-18-12-public.pdf [consultado 2013, 18 de octubre].

Regalado, A. (2012). A Business Report on Digital Education, Harvard Business Review, 116 (1).

Rief, R. (2012). Inaugural Address, [en línea] Disponible en video: http://video. mit.edu/watch/inaugural-address-ofmit-president-l-rafael-reif-12680/ [consultado 2013, 14 de octubre].

Roberts, D.; Greteman, B. (2013). Cloud re-routing, Times Higher Education, [en línea] Disponible en: http://www. timeshighereducation.co.uk/complete-the-digital-learning-revolutiontake-to-the-cloud-say-david-robertsand-blainegreteman/2006498.article [consultado 2013, 15 de octubre].

Santamaría M.; Read T.; Torres D.; Gago, D. (2013). First UNED-CSEV experiences on MOOCs design and dissemination: Balance of results. En: M. Paulsen, F.; Szücs, A. (Eds.). Book of Abstracts, EDEN Conference 2013- Oslo.

Stewart, B. (2013). Massiveness+Openness = New Literacies of Participation, $M E R$ LOT Journal of Online Learning and Teaching, 9 (2), (228-239). 
Siemens, G. (2005). Connectivism: A Learning Theory for the Digital Age, International Journal of Instructional Technology and Distance Learning, 2 (1), (3-10).

Trilling, B.; Fadell, C. (2009). 21st Century Skills: Learning for Life in our times. San Francisco: Jossey-Bass.

Zapata-Ros, M. (2013). MOOCs, una visión crítica y una alternativa complementa- ria: La individualización del aprendizaje y la ayuda pedagógica, Revista Campus Virtuale, 2 (1), (20-38).

Zimmerman, B. (2000). Attaining self-regulation: A social cognitive perspective. En: Boekaerts, M.; Pintrich, P. R.; Zeidner, M. (Eds.). Handbook of self-regulation. San Diego, CA: Academic Press, (13-39).

\section{PERFIL ACADÉMICO Y PROFESIONAL DE LOS AUTORES}

Daniel Torres Mancera. Director General de CSEV. Es conferenciante y panelista habitual en el ámbito nacional e internacional sobre tendencias futuras de la educación y nuevos modelos de aprendizaje y figura clave en el diseño e implementación de algunas de las iniciativas MOOC más destacadas en habla hispana.

E-mail: $\underline{\text { dtorres@csev.org }}$

David Gago Saldaña. Doctor en Economía y Director de Análisis y Prospectiva de la Fundación Centro Superior para la Enseñanza Virtual (CSEV) desde 2011. Es experto en el ámbito de la prospectiva tecnológica aplicada a la educación y en el análisis de sus impactos.

E-mail: dgago@csev.org

DIRECCIÓN DE LOS AUTORES

Fundación Centro Superior para la Enseñanza Virtual (CSEV)

Avenida de Esparta s/n Edificio UNED

28232 - Las Rozas (Madrid)

España

Fecha de recepción: 15/10/2013

Fecha de aceptación: 12/11/2013

\section{Como citar este artículo:}

Torres Mancera, D.; Gago Saldaña, D. (2014). Los MOOCs y su papel en la creación de comunidades de aprendizaje y participación. RIED. Revista Iberoamericana de Educación a Distancia, volumen 17, $\mathrm{n}^{0}$ 1, pp. 13-34. 